\title{
Proof of a conjecture of Z-W Sun on ratio monotonicity
}

Brian Yi Sun ${ }^{*}$, Yingying Hu and Baoyindureng Wu

\section{"Correspondence:}

brianys1984@126.com

College of Mathematics and System

Science, Xinjiang University, Urumqi,

Xinjiang 830046, P.R. China

\begin{abstract}
In this paper, we study the log-behavior of a new sequence $\left\{S_{n}\right\}_{n=0}^{\infty}$, which was defined by Z-W Sun. We find that the sequence is log-convex by using the interlacing method. Additionally, we consider ratio log-behavior of $\left\{S_{n}\right\}_{n=0}^{\infty}$ and find the sequences $\left\{S_{n+1} / S_{n}\right\}_{n=0}^{\infty}$ and $\left\{\sqrt[n]{S_{n}}\right\}_{n=1}^{\infty}$ are log-concave. Our results give an affirmative answer to a conjecture of Z-W Sun on the ratio monotonicity of this new sequence.

MSC: Primary 05A20; 05A10; 11B65; $11 \mathrm{~B} 37$

Keywords: log-convexity; log-concavity; ratio monotonicity; interlacing method; ratio log-concavity
\end{abstract}

\section{Introduction}

Throughout the paper, we denote by $\mathbb{N}$ the set of nonnegative integers. The main objective of this paper aims to confirm a conjecture on ratio monotonicity of a new kind of sequence $\left\{S_{n}\right\}_{n=0}^{\infty}$ via studying its log-behavior properties. The sequence $\left\{S_{n}\right\}_{n=0}^{\infty}$ was introduced by Sun in $[1,2]$ and defined as follows:

$$
S_{n}=\sum_{k=0}^{n}\left(\begin{array}{l}
n \\
k
\end{array}\right)^{2}\left(\begin{array}{c}
2 k \\
k
\end{array}\right)(2 k+1), \quad n \in \mathbb{N} \text {. }
$$

Sun studied congruence and divisibility properties of this kind of numbers in $[1,2]$ and posed the following conjecture.

Conjecture 1.1 ([1], Conjecture 5.2(ii), [2], Conjecture 4.4) The sequence $\left\{\frac{S_{n+1}}{S_{n}}\right\}_{n=3}^{\infty}$ is strictly increasing to the limit 9 , and the sequence $\left\{\frac{\sqrt[n+1]{S_{n+1}}}{\sqrt[n]{S_{n}}}\right\}_{n=1}^{\infty}$ is strictly decreasing to the limit 1 .

To begin with, let us review some related concepts. Let $\left\{z_{n}\right\}_{n=0}^{\infty}$ be a sequence of positive real numbers. We say a sequence $\left\{z_{n}\right\}_{n=0}^{\infty}$ is (strictly) ratio monotonic if its ratio sequence $\left\{\frac{z_{n+1}}{z_{n}}\right\}_{n=0}^{\infty}$ is (strictly) monotonically increasing or (strictly) decreasing as $n$ increases. A sequence $\left\{z_{n}\right\}_{n=0}^{\infty}$ is said to be log-convex (resp. log-concave) if, for all $n \geq 1$,

$$
z_{n-1} z_{n+1} \geq z_{n}^{2} \quad\left(\text { resp. } z_{n-1} z_{n+1} \leq z_{n}^{2}\right)
$$

(c) Sun et al. 2016. This article is distributed under the terms of the Creative Commons Attribution 4.0 International License (http://creativecommons.org/licenses/by/4.0/), which permits unrestricted use, distribution, and reproduction in any medium, provided you give appropriate credit to the original author(s) and the source, provide a link to the Creative Commons license, and indicate if changes were made. 
Meanwhile, the sequence $\left\{z_{n}\right\}_{n=0}^{\infty}$ is called strictly log-convex (resp. log-concave) if the inequality in (1.2) is strict for $n \geq n_{0}$ for some $n_{0} \in \mathbb{N}$. Indeed, ratio monotonicity is equivalent to log-behavior. According to the definitions, it is easy to see that a ratio monotonically increasing (resp. decreasing) sequence is itself a log-convex (resp. log-concave) sequence and vice versa.

So far, many criteria for log-behavior of a sequence have been developed; see [3-10] and the references therein for details. Also, there have been some important progress on ratio monotonicity since many conjectures on ratio monotonicity were posed by Sun [11]. For example, the reader may refer to [12-14]. Recently, Chen et al. [15] introduced a notion called ratio log-behavior in order to study the log-behavior of sequences of the form $\left\{\sqrt[n]{z_{n}}\right\}_{n=1}^{\infty}$. By ratio log-concavity (resp. log-convexity) of a sequence $\left\{z_{n}\right\}_{n=0}^{\infty}$, we mean that the sequence $\left\{\frac{z_{n+1}}{z_{n}}\right\}_{n=0}^{\infty}$ is log-concave (resp. log-convex). They found that the ratio logconcavity (resp. log-convexity) of a positive sequence $\left\{z_{n}\right\}_{n=k_{0}}^{\infty}$ for some positive integer $k_{0}$ can imply the sequence $\left\{\sqrt[n]{z_{n}}\right\}_{n=k_{0}}^{\infty}$ is strictly log-concave (resp. log-convex) if it satisfies certain initial conditions; see [15], Theorem 3.1 and Theorem 3.6. To make this paper self-contained, we will recall their criteria in Section 3.

The main results of the present paper can be stated as follows.

Theorem 1.2 The sequence $\left\{S_{n}\right\}_{n=0}^{\infty}$ is strictly log-convex, that is,

$$
S_{n}^{2}<S_{n+1} S_{n-1} \quad \text { for } n \geq 1 .
$$

Theorem 1.3 The sequence $\left\{S_{n}\right\}_{n=0}^{\infty}$ is ratio log-concave, that is,

$$
\left(\frac{S_{n+1}}{S_{n}}\right)^{2} \geq \frac{S_{n}}{S_{n-1}} \cdot \frac{S_{n+2}}{S_{n+1}} \text { for } n \geq 0
$$

Theorem 1.4 The sequence $\left\{\sqrt[n]{S_{n}}\right\}_{n=1}^{\infty}$ is strictly log-concave.

On the basis of Theorem 1.2 and Theorem 1.4, we can conclude the following result.

Theorem 1.5 Conjecture 1.1 is true.

The remainder of the paper is organized as follows. We give some preliminaries work in Section 2, including a three-term recurrence for $S_{n}$, a lower and upper bound for $\frac{S_{n}}{S_{n-1}}$. In Section 3, we give proofs of our main theorems.

\section{Preliminaries}

\subsection{A three-term recurrence}

The Zeilberger algorithm [16] yields the following four-term recurrence for $S_{n}$ :

$$
\begin{aligned}
& 9(n+1)^{2} S_{n}-\left(19 n^{2}+74 n+87\right) S_{n+1}+(n+3)(11 n+29) S_{n+2} \\
& -(n+3)^{2} S_{n+3}=0 .
\end{aligned}
$$

This recurrence cannot easily be tackled as almost all criteria for log-behavior are concerned with three-term recurrences. So it is indispensable for us to find a three-term recurrence. The following lemma was first obtained awkwardly by solving a linear system 
of equations. Afterwards, we found it can be deduced from a three-term recurrence for $4 n S_{n}$, which was established by a technique way due to Guo and Liu [17].

Lemma 2.1 Let $S_{n}$ be defined in (1.1). Then it satisfies a three-term recurrence:

$$
\begin{aligned}
& (n+1)^{2}(4 n-1)(4 n+3) S_{n+1}-(4 n-1)(4 n+7)\left(10 n^{2}+10 n+3\right) S_{n} \\
& \quad+9 n^{2}(4 n+3)(4 n+7) S_{n-1}=0 \quad \text { for } n \geq 1 .
\end{aligned}
$$

Proof Let $u_{n}=4 n S_{n}$. Guo and Liu [17], Eq. (2.4), found a three-term recurrence for $u_{n}$, i.e.,

$$
\begin{aligned}
& n(n+1)(n+2)(4 n+3)(4 n+7) u_{n+2}-n(4 n+3)(4 n+11)\left(10 n^{2}+30 n+23\right) u_{n+1} \\
& \quad+9(n+1)^{3}(4 n+11)(4 n+7) u_{n}=0 .
\end{aligned}
$$

Substituting $4 n S_{n}$ for $u_{n}$ in (2.2) and then simplifying, the recurrence (2.1) follows easily.

\subsection{Bounds for $\frac{s_{n}}{S_{n-1}}$}

In [4], Chen and Xia provided a heuristic approach to find bounds for $\frac{z_{n}}{z_{n-1}}$, where $z_{n}$ satisfies a three-term recurrence. The following bounds can be acquired by using their method.

\section{Lemma 2.2 Let}

$$
h(n)=9-\frac{9}{2 n^{2}} .
$$

Then we have

$$
h(n-1)<\frac{S_{n}}{S_{n-1}}<h(n) \quad \text { for } n \geq 2 .
$$

Proof We proceed our proof by induction on $n$. For the sake of simplicity, let

$$
s_{n}=\frac{S_{n}}{S_{n-1}}
$$

To begin with,

$$
h(1)=\frac{9}{2}<s_{2}=\frac{55}{7}<h(2)=\frac{63}{8},
$$

so inequality (2.3) holds for $n=2$.

Suppose that $h(n-1)<s_{n}<h(n)$, we proceed to show that $h(n)<s_{n+1}<h(n+1)$.

On the one hand, by Lemma 2.1, we have

$$
\begin{aligned}
s_{n+1}-h(n+1) & =\frac{(4 n+7)\left(10 n^{2}+10 n+3\right)}{(n+1)^{2}(4 n+3)}-\frac{9 n^{2}(4 n+7)}{(n+1)^{2}(4 n-1) s_{n}}-h(n+1) \\
& <\frac{4 n+7}{(n+1)^{2}}\left(\frac{10 n^{2}+10 n+3}{4 n+3}-\frac{9 n^{2}}{(4 n-1) h(n)}\right)-h(n+1)
\end{aligned}
$$




$$
\begin{aligned}
& =\frac{4 n+7}{(n+1)^{2}}\left(\frac{72 n^{5}+54 n^{4}-36 n^{3}-36 n^{2}-2 n+3}{(4 n-1)(4 n+3)\left(2 n^{2}-1\right)}\right)-h(n+1) \\
& =\frac{-88 n^{2}-40 n+15}{2(n+1)^{2}(4 n-1)(4 n+3)\left(2 n^{2}-1\right)} \\
& <0 \quad \text { for } n \geq 1,
\end{aligned}
$$

which obviously implies $s_{n+1}<h(n+1)$.

On the other hand, consider that, for $n \geq 1$,

$$
\begin{aligned}
s_{n+1}-h(n) & =\frac{(4 n+7)\left(10 n^{2}+10 n+3\right)}{(n+1)^{2}(4 n+3)}-\frac{9 n^{2}(4 n+7)}{(n+1)^{2}(4 n-1) s_{n}}-h(n) \\
& >\frac{4 n+7}{(n+1)^{2}}\left(\frac{10 n^{2}+10 n+3}{4 n+3}-\frac{9 n^{2}}{(4 n-1) h(n-1)}\right)-h(n) \\
& =\frac{(4 n+7)\left(72 n^{5}-90 n^{4}-72 n^{3}+10 n^{2}+14 n-3\right)}{(n+1)^{2}(4 n-1)(4 n+3)\left(2 n^{2}-4 n+1\right)}-h(n) \\
& =\frac{512 n^{5}-792 n^{4}-728 n^{3}+147 n^{2}+126 n-27}{2 n^{2}(n+1)^{2}(4 n-1)(4 n+3)\left(2 n^{2}-4 n+1\right)} \\
& >0 .
\end{aligned}
$$

Evidently, this gives us $s_{n+1}>h(n)$.

According to an inductive argument, it follows that, for all $n \geq 2$, we have

$$
h(n-1)<s_{n}<h(n)
$$

As a corollary, we have the following.

Corollary 2.3 Let $S_{n}$ be defined by (1.1). Then we have

$$
\lim _{n \rightarrow \infty} \frac{S_{n+1}}{S_{n}}=9 .
$$

\section{Proofs of theorems}

Before giving proofs of our theorems, we need to recall some known results. The following proposition first appeared in [6] and is formally called the interlacing method by Došlić and Veljan [5].

Proposition 3.1 ([5]) Suppose that $\left\{z_{n}\right\}_{n=0}^{\infty}$ is a sequence of positive numbers. Then, for some positive integer $N$, the sequence $\left\{z_{n}\right\}_{n=N}^{\infty}$ is log-convex (resp.log-concave) if there exists an increasing (resp. a decreasing) sequence $\{h(n)\}_{n=0}^{\infty}$ such that

$$
h(n-1) \leq q_{n} \leq h(n) \quad\left(\text { resp. } h(n-1) \geq q_{n} \geq h(n)\right)
$$

holds for $n \geq N+1$, where $q_{n}=\frac{z_{n+1}}{z_{n}}$. Moreover, the sequence $\left\{z_{n}\right\}_{n=N}^{\infty}$ is strictly log-convex (resp. strictly log-concave) if and only if the above inequalities (3.1) are strict.

To prove Theorem 1.3 and Theorem 1.4, the following criteria due to Chen et al. [15] are also indispensable. 
Theorem 3.2 ([15], Theorem 3.1) Let $\left\{z_{n}\right\}_{n=0}^{\infty}$ be the sequence defined by the following recurrence:

$$
z_{n}=u(n) z_{n-1}+v(n) z_{n-2}
$$

Assume that $v(n)<0$ for $n \geq 2$. If there exist a nonnegative integer $N$ and a function $h(n)$ such that, for all $n \geq N+2$,

(i) $\frac{3 u(n)}{4} \leq \frac{z_{n}}{z_{n-1}} \leq h(n)$;

(ii) $h(n)^{4}-u(n) h(n)^{3}-u(n+1) v(n) h(n)-v(n) v(n+1)<0$,

then $\left\{z_{n}\right\}_{n=N}^{\infty}$ is ratio log-concave.

Theorem 3.3 ([15], Theorem 3.6) Assume that $k$ is a positive integer. If a sequence $\left\{z_{n}\right\}_{n=k}^{\infty}$ is ratio log-concave and

$$
\frac{\sqrt[k+1]{z_{k+1}}}{\sqrt[k]{z_{k}}}>\frac{\sqrt[k+2]{z_{k+2}}}{\sqrt[k+1]{z_{k+1}}}
$$

then the sequence $\left\{\sqrt[n]{z_{n}}\right\}_{n=k}^{\infty}$ is strictly log-concave.

We are now in a position to prove our main theorems.

Proof of Theorem 1.2 Since $h(n)$ is strictly monotonically increasing, it follows that $\left\{S_{n}\right\}_{n=1}^{\infty}$ is strictly log-convex by Lemma 2.2 and Proposition 3.1.

As a corollary, we have the following.

Corollary 3.4 The sequence $\left\{\frac{S_{n+1}}{S_{n}}\right\}_{n=1}^{\infty}$ is strictly monotonically increasing.

Since $\left\{S_{n}\right\}_{n=0}^{\infty}$ is a positive sequence, we can define the sequence $\left\{\sqrt[n]{S_{n}}\right\}_{n=1}^{\infty}$. Then we have the following result.

Corollary 3.5 The sequence $\left\{\sqrt[n]{S_{n}}\right\}_{n=1}^{\infty}$ is strictly increasing. Moreover,

$$
\lim _{n \rightarrow \infty} \sqrt[n]{S_{n}}=9
$$

Proof By Corollary 3.4, it follows that

$$
\frac{S_{n+1}}{S_{n}}>\frac{S_{n}}{S_{n-1}} \text { for } n \geq 1 .
$$

Consider that $S_{0}=1$, so

$$
S_{n}=S_{0} \cdot \frac{S_{1}}{S_{0}} \cdot \frac{S_{2}}{S_{1}} \cdots \frac{S_{n}}{S_{n-1}}<\left(\frac{S_{n+1}}{S_{n}}\right)^{n},
$$

which implies

$$
S_{n}^{n+1}<S_{n+1}^{n} .
$$


This is equivalent to

$$
\left(S_{n}^{n+1}\right)^{\frac{1}{n(n+1)}}<\left(S_{n+1}^{n}\right)^{\frac{1}{n(n+1)}}
$$

that is,

$$
\sqrt[n]{S_{n}}<\sqrt[n+1]{S_{n+1}}
$$

Additionally, consider that, for a real sequence $\left\{z_{n}\right\}_{n=1}^{\infty}$ of positive real numbers, it was shown that

$$
\lim _{n \rightarrow \infty} \inf \frac{z_{n+1}}{z_{n}} \leq \lim _{n \rightarrow \infty} \inf \sqrt[n]{z_{n}}
$$

and

$$
\lim _{n \rightarrow \infty} \sup \sqrt[n]{z_{n}} \leq \lim _{n \rightarrow \infty} \sup \frac{z_{n+1}}{z_{n}}
$$

see Rudin [18]. The inequalities in (3.3) and (3.4) imply that

$$
\lim _{n \rightarrow \infty} \sqrt[n]{z_{n}}=\lim _{n \rightarrow \infty} \frac{z_{n}}{z_{n-1}}
$$

if $\lim _{n \rightarrow \infty} \frac{z_{n}}{z_{n-1}}$ exists. By Corollary 2.3, we arrive at (3.2).

This completes the proof.

Proof of Theorem 1.3 By Lemma 2.1, the recurrence (2.1) implies that

$$
S_{n}=\frac{(4 n+3)\left(10 n^{2}-10 n+3\right)}{n^{2}(4 n-1)} S_{n-1}-\frac{9(n-1)^{2}(4 n+3)}{n^{2}(4 n-5)} S_{n-1} .
$$

To keep the notation in Theorem 3.2, here we still let

$$
u(n)=\frac{(4 n+3)\left(10 n^{2}-10 n+3\right)}{n^{2}(4 n-1)}, \quad v(n)=\frac{9(n-1)^{2}(4 n+3)}{n^{2}(4 n-5)}
$$

Consider that

$$
\begin{aligned}
\frac{3 u(n)}{4}-h(n-1) & =-\frac{3\left(8 n^{5}-18 n^{4}+6 n^{3}-41 n^{2}+36 n-9\right)}{4 n^{2}(n-1)^{2}(4 n-1)} \\
& <0 \quad \text { for } n \geq 3,
\end{aligned}
$$

which shows that

$$
\frac{3 u(n)}{4}<h(n-1) \text { for } n \geq 3 .
$$

Additionally, for $n \geq 1$,

$$
h(n)^{4}-u(n) h(n)^{3}-u(n+1) v(n) h(n)-v(n) v(n+1)
$$




$$
\begin{aligned}
& =\frac{-A(n)}{16 n^{8}(n+1)^{2}(4 n-5)(4 n-1)} \\
& <0,
\end{aligned}
$$

where

$$
\begin{aligned}
A(n)= & 331,776 n^{8}-393,984 n^{7}-693,360 n^{6}+524,232 n^{5}+581,256 n^{4}-242,028 n^{3} \\
& -223,803 n^{2}+39,366 n+32,805 .
\end{aligned}
$$

Combining the inequalities (3.5) and (3.6), we arrive at our statement in Theorem 1.3 by Theorem 3.2.

Remark 3.6 Notice that the first author of the present paper and Zhao [19] also found some criteria for ratio log-behavior, which can also be used to prove ratio log-concavity of $\left\{S_{n}\right\}_{n=0}^{\infty}$.

Proof of Theorem 1.4 By Theorem 3.3, it suffices to find a positive integer $k$ such that

$$
\frac{\sqrt[k+1]{S_{k+1}}}{\sqrt[k]{S_{k}}}>\frac{\sqrt[k+2]{S_{k+2}}}{\sqrt[k+1]{S_{k+1}}}
$$

Let $k=1$, we have

$$
\frac{\sqrt{S_{2}}}{S_{1}}=\frac{\sqrt{55}}{7}>\frac{\sqrt{S_{3}}}{S_{2}}=\frac{\sqrt[3]{93}}{\sqrt[6]{5} \sqrt{11}}
$$

since

$$
\left(\frac{\sqrt{55}}{7}\right)^{6}-\left(\frac{\sqrt[3]{93}}{\sqrt[6]{5} \sqrt{11}}\right)^{6}=\frac{89,679,424}{782,954,095}>0
$$

Therefore, by Theorem 1.3 and Theorem 3.3, it follows that $\left\{\sqrt[n]{S_{n}}\right\}_{n=1}^{\infty}$ is strictly logconcave.

Corollary 3.7 The sequence $\left\{\frac{\sqrt[n+1]{S_{n+1}}}{\sqrt[n]{S_{n}}}\right\}_{n=1}^{\infty}$ is strictly monotonically decreasing.

Corollary 3.8 For $S_{n}$, we have

$$
\lim _{n \rightarrow \infty} \frac{\sqrt[n+1]{S_{n+1}}}{\sqrt[n]{S_{n}}}=1
$$

Proof With the aid of Lemma 2.2, we have

$$
7 \prod_{i=2}^{n} h(i-1)<S_{n}<7 \prod_{i=2}^{n} h(i) .
$$

Therefore, we can deduce that

$$
\log \left(\frac{\sqrt[n+1]{S_{n+1}}}{\sqrt[n]{S_{n}}}\right)=\frac{\log S_{n+1}}{n+1}-\frac{\log S_{n}}{n}
$$




$$
<\frac{\log \left(7 \prod_{i=2}^{n+1} h(i)\right)}{n+1}-\frac{\log \left(7 \prod_{i=2}^{n} h(i-1)\right)}{n}
$$

and

$$
\begin{aligned}
\log \left(\frac{\sqrt[n+1]{S_{n+1}}}{\sqrt[n]{S_{n}}}\right) & =\frac{\log S_{n+1}}{n+1}-\frac{\log S_{n}}{n} \\
& >\frac{\log \left(7 \prod_{i=2}^{n+1} h(i-1)\right)}{n+1}-\frac{\log \left(7 \prod_{i=2}^{n} h(i)\right)}{n} .
\end{aligned}
$$

Resorting to Mathematica 10.0 , we find that

$$
\begin{aligned}
& \lim _{n \rightarrow \infty}\left(\frac{\log \left(7 \prod_{i=2}^{n+1} h(i)\right)}{n+1}-\frac{\log \left(7 \prod_{i=2}^{n} h(i-1)\right)}{n}\right)=0, \\
& \lim _{n \rightarrow \infty}\left(\frac{\log \left(7 \prod_{i=2}^{n+1} h(i-1)\right)}{n+1}-\frac{\log \left(7 \prod_{i=2}^{n} h(i)\right)}{n}\right)=0 .
\end{aligned}
$$

Thus we can arrive at

$$
\lim _{n \rightarrow \infty} \log \left(\frac{\sqrt[n+1]{S_{n+1}}}{\sqrt[n]{S_{n}}}\right)=0
$$

which implies

$$
\lim _{n \rightarrow \infty} \frac{\sqrt[n+1]{S_{n+1}}}{\sqrt[n]{S_{n}}}=1
$$

This finishes the proof.

Proof of Theorem 1.5 The first part of Conjecture 1.1 follows from Theorem 1.2, Corollary 2.3 and Corollary 3.4. The second part follows from Theorem 1.4, Corollary 3.7, and Corollary 3.8. This completes the proof of Theorem 1.5.

\section{Competing interests}

The authors declare that they have no competing interests.

\section{Authors' contributions}

All authors contributed equally to the writing of this paper. All authors read and approved the final manuscript.

\section{Acknowledgements}

We would like to thank the Editor, the Associate Editor and the anonymous referees for their careful reading and constructive comments which have helped us to significantly improve the presentation of the paper. The first two authors was supported by the Scientific Research Program of the Higher Education Institution of Xinjiang Uygur Autonomous Region (No. XJEDU2016S032) and the third author was supported by NSFC (No. 11571294).

Received: 5 August 2016 Accepted: 25 October 2016 Published online: 04 November 2016

\section{References}

1. Sun, ZW: Two new kinds of numbers and related divisibility results. Preprint. arXiv:1408.5381

2. Sun, ZW: Two new kinds of numbers and their arithmetic properties. Preprint. arXiv:1408.5381v2

3. Brenti, F: Log-concave and unimodal sequences in algebra, combinatorics and geometry: an update. In: Jerusalem Combinatorics '93. Contemp. Math., vol. 178, pp. 71-89 (1994)

4. Chen, WYC, Xia, EXW: The 2-log-convexity of the Apéry numbers. Proc. Am. Math. Soc. 139(2), 391-400 (2011)

5. Došlić, T, Veljan, D: Logarithmic behavior of some combinatorial sequences. Discrete Math. 308, 2182-2212 (2008)

6. Liu, LL, Wang, Y: On the log-convexity of combinatorial sequences. Adv. Appl. Math. 39(4), 453-476 (2007)

7. Stanley, RP: Log-concave and unimodal sequences in algebra, combinatorics and geometry. In: Graph Theory and Its Applications: East and West (Jinan, 1986). Ann. New York Acad. Sci., vol. 576, pp. 500-535. N.Y. Acad. Sci., New York (1989) 
8. Sun, BY, Wu, B: Two-log-convexity of the Catalan-Larcombe-French sequence. J. Inequal. Appl. 2015, 404 (2015)

9. Xia, EXW, Yao, OXM: A criterion for the log-convexity of combinatorial sequences. Electron. J. Comb. 20(4), paper 3 (2013)

10. Yang, ALB, Zhao, JJY: Log-concavity of the Fennessey-Larcombe-French sequence. Taiwan. J. Math. 20(5), 993-999 (2016)

11. Sun, ZW: Conjectures involving arithmetical sequences. In: Kanemitsu, S, Li, H, Liu, J (eds.) Numbers Theory: Arithmetic in Shangri-La, Proc. 6th China-Japan Seminar (Shanghai, August 15-17, 2011), pp. 244-258. World Scientific, Singapore (2013)

12. Hou, QH, Sun, ZW, Wen, HM: On monotonicity of some combinatorial sequences. Publ. Math. (Debr.) 85, 285-295 (2014)

13. Luca, F, Stănică, P: On some conjectures on the monotonicity of some combinatorial sequences. J. Comb. Number Theory 4, 1-10 (2012)

14. Wang, Y, Zhu, BX: Proofs of some conjectures on monotonicity of number-theoretic and combinatorial sequences. Sci. China Math. 57, 2429-2435 (2014)

15. Chen, WYC, Guo, JJF, Wang, LXW: Infinitely log-monotonic combinatorial sequences. Adv. Appl. Math. 52, 99-120 (2014)

16. Zeilberger, D: The method of creative telescoping. J. Symb. Comput. 11, 195-204 (1991)

17. Guo, VJW, Liu, JC: Proof of some conjectures of Z.-W. Sun on the divisibility of certain double-sums. Int. J. Number Theory 12(3), 615-623 (2016)

18. Rudin, W: Principles of Mathematical Analysis, 3rd edn. McGraw-Hill, New York (2004)

19. Sun, BY, Zhao, JJY: Log-behavior of two sequences related to the elliptic integrals. Preprint. arXiv:1602.04359

\section{Submit your manuscript to a SpringerOpen ${ }^{\ominus}$ journal and benefit from:}

- Convenient online submission

Rigorous peer review

- Immediate publication on acceptance

- Open access: articles freely available online

- High visibility within the field

- Retaining the copyright to your article 